\title{
Lattice Regularization and Symmetries
}

\author{
Peter Hasenfratz, Ferenc Niedermayer* and Reto von Allmen \\ Institute for Theoretical Physics \\ University of Bern \\ Sidlerstrasse 5, CH-3012 Bern, Switzerland
}

\begin{abstract}
Finding the relation between the symmetry transformations in the continuum and on the lattice might be a nontrivial task as illustrated by the history of chiral symmetry. Lattice actions induced by a renormalization group procedure inherit all symmetries of the continuum theory. We give a general procedure which gives the corresponding symmetry transformations on the lattice.
\end{abstract}

* On leave from Eötvös University, HAS Research Group, Budapest, Hungary 


\section{Introduction}

In 1981 Ginsparg and Wilson formulated a condition [1] to be satisfied by the lattice Dirac operator in order to have the physical consequences of chiral symmetry on the lattice. The derivation of this condition is based on renormalization group (RG) considerations, but the result is more general. Indeed, the GW relation is satisfied not only by the fixed point [2, but also by the overlap $[3$ and the domain-wall operator after dimensional reduction [4]. The Dirac operators in the latter two cases are not related to RG ideas.

The GW condition is a non-linear relation for the lattice Dirac operator reflecting the fact that concerning chiral symmetry the physical content of the classical lattice theory is the same as that in the continuum. It was observed only many years later that an exact symmetry transformation exists on the lattice as well [5].

Discretizing a field theory by repeated RG block transformation - or equivalently, by 'blocking out of continuum' 6 - has the advantage that all symmetries of the continuum theory will be inherited by the lattice action - even those which are explicitly broken by the block transformation. The symmetry transformations are, however, different from those in the continuum. We present here a general technique and a streamlined procedure to find the form of the symmetry transformations and the symmetry conditions (like the GW relation).

We have to emphasize that talking about a symmetry transformation on the lattice we mean a symmetry of the lattice action, i.e. the classical field theory. In the quantum theory this transformation enters as a change of variable in the path integral which might induce a non-trivial contribution to the Ward identity by the integration measure.

Not all internal symmetries of the continuum theory can be kept by the block transformation. Consider, as an example, the chiral symmetry. For a continuum action and a block transformation which both have an explicit $\gamma_{5^{-}}$ invariance, the resulting lattice action will also be $\gamma_{5}$-invariant. But due to the existence of the chiral anomaly, this action cannot be an acceptable one - it has to be either non-local or has to describe extra unwanted degrees of freedom (doublers) [7. This is what happens in the limit when the coefficient of the natural block transformation goes to infinity. In this limit the blocking becomes chiral invariant, but at the same time the corresponding lattice action ceases to be local [8].

This paper is motivated by some unsolved theoretical problems in lattice regularized chiral gauge theories. In spite of the great progress during the last years [9] an important problem remains: the relative weight between the different topological sectors is undefined. This situation might be related to 
different technical problems. Although the chiral invariant vector theory has a controlled RG background, the steps towards a chiral theory are not related to RG anymore. The projectors [10] are introduced by hand and, seemingly unavoidably, they break CP and T symmetry 11. Further, the fermion number anomaly ${ }^{1}$ enters in an unusual way: the different topological sectors have different number of degrees of freedom on the lattice. These technical issues might be related to the problem mentioned above. A different strategy would be to start with a fermion number violating block transformation, which makes the relation between the continuum and lattice symmetries non-trivial. The systematic approach discussed here might be a useful tool in this and similar problems.

\section{Free massless fermions}

Since fermions enter quadratically even in the presence of gauge fields, most of the equations below remain valid in the presence of interactions as well.

The block transformation is a Gaussian integral which is equivalent to a formal minimization problem:

$$
\bar{\chi} \mathcal{D} \chi=\min _{\bar{\psi}, \psi}\left\{\bar{\psi} D \psi+\left(\bar{\chi}-\bar{\psi} \omega^{\dagger}\right)(\chi-\omega \psi)\right\}
$$

where the fermion fields $\psi_{x}$ and $\chi_{n}$ live in the continuum and on the lattice respectively, $\omega_{n x}$ is the blocking matrix, $D_{x x^{\prime}}=\left(\gamma^{\mu} \partial_{\mu}\right)_{x x^{\prime}}$ and $\mathcal{D}_{n n^{\prime}}$ are the continuum and lattice Dirac operators. For the blocking we take a flat, nonoverlapping averaging

$$
\omega_{n x}= \begin{cases}1 & \text { if } x \in \text { block } n \\ 0 & \text { otherwise }\end{cases}
$$

With this choice one has $\sum_{x} \omega_{n x} \omega_{x n^{\prime}}^{\dagger}=\delta_{n n^{\prime}}$, i.e. $\omega \omega^{\dagger}=1$.

The minimizing fields $\psi_{0}=\psi_{0}(\chi)$ and $\bar{\psi}_{0}=\bar{\psi}_{0}(\bar{\chi})$ in eq. (1) are given by

$$
\begin{aligned}
\psi_{0}(\chi) & =A^{-1} \omega^{\dagger} \chi \\
\bar{\psi}_{0}(\bar{\chi}) & =\bar{\chi} \omega A^{-1}
\end{aligned}
$$

where

$$
A=D+\omega^{\dagger} \omega
$$

Inserting eq. (3) into eq. (1) gives the lattice Dirac operator

$$
\mathcal{D}=1-\omega A^{-1} \omega^{\dagger} .
$$

\footnotetext{
${ }^{1}$ We mean here the global vector anomaly of a chiral gauge theory free of gauge anomalies.
} 
From the equations above it is easy to derive the following useful relations which will be used repeatedly in this work

$$
\begin{array}{lll}
\omega \psi_{0}(\chi) & =(1-\mathcal{D}) \chi, & \bar{\psi}_{0}(\bar{\chi}) \omega^{\dagger}=\bar{\chi}(1-\mathcal{D}), \\
D \psi_{0}(\chi)=\omega^{\dagger} \mathcal{D} \chi, & \bar{\psi}_{0}(\bar{\chi}) D=\bar{\chi} \mathcal{D} \omega .
\end{array}
$$

The Ginsparg-Wilson relation can be obtained then from eq. (5) by using $\left\{D, \gamma_{5}\right\}=0$ and the relations above ${ }^{2}$ :

$$
\left\{\mathcal{D}, \gamma_{5}\right\}=2 \mathcal{D} \gamma_{5} \mathcal{D}
$$

We formulate now a general statement on the form of infinitesimal symmetry transformations on the lattice.

\section{Statement}

Let $\delta \psi$ and $\delta \bar{\psi}$ be the change of the corresponding continuum fields under an infinitesimal symmetry transformation which leaves invariant the continuum action $\bar{\psi} D \psi$.

Define the infinitesimal change of the lattice fields by

$$
\delta \chi=\omega \delta \psi_{0}(\chi), \quad \delta \bar{\chi}=\delta \bar{\psi}_{0}(\bar{\chi}) \omega^{\dagger} .
$$

Then the lattice action $\bar{\chi} \mathcal{D} \chi$ is invariant under this infinitesimal symmetry transformations.

Proof

One can use the explicit equations above to show the statement.

Replace $\psi_{0}(\chi)$ by $\psi_{0}(\chi+\delta \chi)-\delta \psi_{0}(\chi)$ and $\bar{\psi}_{0}(\bar{\chi})$ by $\bar{\psi}_{0}(\bar{\chi}+\delta \bar{\chi})-\delta \bar{\psi}_{0}(\bar{\chi})$ on the r.h.s of eq. (1), where $\delta \psi_{0}(\chi)$ is an infinitesimal continuum symmetry transformation of $\psi_{0}(\chi)$ and $\delta \chi$ is a not yet defined infinitesimal change of $\chi$. Since $\psi_{0}(\chi+\delta \chi)-\delta \psi_{0}(\chi)=\psi_{0}(\chi)+$ infinitesimallysmall and $\psi_{0}(\chi)$ is the minimum of the r.h.s. of eq. (1), the change of the r.h.s. is quadratically small:

$$
\begin{aligned}
& \bar{\chi} \mathcal{D} \chi=\bar{\psi}_{0}(\bar{\chi}+\delta \bar{\chi}) D \psi_{0}(\chi+\delta \chi)+ \\
& \quad\left[( \overline { \chi } - ( \overline { \psi } _ { 0 } ( \overline { \chi } + \delta \overline { \chi } ) - \delta \overline { \psi } _ { 0 } ( \overline { \chi } ) ) \omega ^ { \dagger } ] \left[\left(\chi-\omega\left(\psi_{0}(\chi+\delta \chi)-\delta \psi_{0}(\chi)\right)\right]+\right.\right. \\
& \quad \text { quadratically small }
\end{aligned}
$$

where, in the first term on the r.h.s. of eq. (9), we used that $\delta \psi_{0}(\chi)$ is a symmetry transformation in the continuum.

We identify now

$$
\delta \chi=\omega \delta \psi_{0}(\chi), \quad \delta \bar{\chi}=\delta \bar{\psi}_{0}(\bar{\chi}) \omega^{\dagger},
$$

\footnotetext{
${ }^{2}$ Note that with our choice of the coefficients in eq. (1) the factor 2 appears in the GW relation. This is, of course, just a convention.
} 
which leads to

$$
\begin{aligned}
\bar{\chi} \mathcal{D} \chi= & \bar{\psi}_{0}(\bar{\chi}+\delta \bar{\chi}) D \psi_{0}(\chi+\delta \chi)+ \\
& +\left[\bar{\chi}+\delta \bar{\chi}-\bar{\psi}_{0}(\bar{\chi}+\delta \bar{\chi}) \omega^{\dagger}\right]\left[\chi+\delta \chi-\omega \psi_{0}(\chi+\delta \chi)\right]
\end{aligned}
$$

up to quadratically small corrections. Comparing eq. (11) and eq. (11) we obtain

$$
(\bar{\chi}+\delta \bar{\chi}) \mathcal{D}(\chi+\delta \chi)=\bar{\chi} \mathcal{D} \chi
$$

i.e. eq. (10) is a symmetry transformation of the lattice action $\bar{\chi} \mathcal{D} \chi$.

\section{Symmetry transformations of the lattice ac- tion, examples}

\section{$U(1)$ axial transformation}

The standard infinitesimal axial rotation in the continuum reads

$$
\delta \psi_{0}(\chi)=\mathrm{i} \epsilon \gamma_{5} \psi_{0}(\chi), \quad \delta \bar{\psi}_{0}(\bar{\chi})=\mathrm{i} \epsilon \bar{\psi}_{0}(\bar{\chi}) \gamma_{5} .
$$

The corresponding lattice transformation has the form

$$
\begin{aligned}
& \delta \chi=\mathrm{i} \epsilon \gamma_{5} \omega \psi_{0}(\chi)=\mathrm{i} \epsilon \gamma_{5}(1-\mathcal{D}) \chi, \\
& \delta \bar{\chi}=\mathrm{i} \epsilon \bar{\psi}_{0}(\bar{\chi}) \gamma_{5} \omega^{\dagger}=\mathrm{i} \epsilon \bar{\chi}(1-\mathcal{D}) \gamma_{5},
\end{aligned}
$$

where we used eq. (8) and eq. (6). These transformations have the well known form found by Lüscher [5]. Notice, however that the axial transformation in the continuum is not unique. The following transformation, for example, also leaves the continuum action invariant

$$
\delta \psi_{0}(\chi)=\mathrm{i} \epsilon \gamma_{5}(1-\alpha D) \psi_{0}(\chi), \quad \delta \bar{\psi}_{0}(\bar{\chi})=\mathrm{i} \epsilon \bar{\psi}_{0}(\bar{\chi})(1+\alpha D) \gamma_{5} .
$$

The associated lattice transformation reads

$$
\begin{aligned}
& \delta \chi=\mathrm{i} \epsilon \gamma_{5}(1-(1+\alpha) \mathcal{D}) \chi \\
& \delta \bar{\chi}=\mathrm{i} \epsilon \bar{\chi}(1-(1-\alpha) \mathcal{D}) \gamma_{5} .
\end{aligned}
$$

The $\alpha=1$ case $^{3}$ is special since $\gamma_{5}$ and $\hat{\gamma}_{5}=\gamma_{5}(1-2 \mathcal{D})$, (for which $\hat{\gamma}_{5}^{2}=1$ ) are candidates to build the lattice $\mathrm{L} / \mathrm{R}$ projectors for $\bar{\chi}$ and $\chi$, respectively [10. Notice the asymmetry between the transformations of $\chi$ and $\bar{\chi}$, which is the source of $\mathrm{CP}$ violation in the present formulation of chiral gauge gauge theories mentioned in the introduction.

\footnotetext{
${ }^{3}$ as well as the $\alpha=-1$ case
} 
$U(1)$ vector transformation

The standard infinitesimal vector rotation in the continuum $\delta \psi_{0}(\chi)=\mathrm{i} \epsilon \psi_{0}(\chi)$, $\delta \bar{\psi}_{0}(\bar{\chi})=-\mathrm{i} \epsilon \bar{\psi}_{0}(\bar{\chi})$ implies the lattice transformation

$$
\delta \chi=\mathrm{i} \epsilon(1-\mathcal{D}) \chi, \quad \delta \bar{\chi}=-\mathrm{i} \epsilon \bar{\chi}(1-\mathcal{D})
$$

while the transformation $\delta \psi_{0}(\chi)=\mathrm{i} \epsilon(1-\alpha D) \psi_{0}(\chi), \delta \bar{\psi}_{0}(\bar{\chi})=-\mathrm{i} \epsilon \bar{\psi}_{0}(\bar{\chi})(1-\alpha D)$ leads to

$$
\begin{aligned}
& \delta \chi=\mathrm{i} \epsilon(1-(1+\alpha) \mathcal{D}) \chi \\
& \delta \bar{\chi}=-\mathrm{i} \epsilon \bar{\chi}(1-(1+\alpha) \mathcal{D}) .
\end{aligned}
$$

For $\alpha=0$ the continuum, while for $\alpha=-1$ the lattice transformation has the standard form.

Considering finite transformations, note that for $\alpha=0$ in eqs. (15), (16) the transformation $\exp \left(i t \gamma_{5}(1-\mathcal{D})\right)$ is not compact (it is not $2 \pi$-periodic in $t$ ) as opposed to the corresponding transformation $\exp \left(i t \gamma_{5}\right)$ in the continuum. On the other hand, for $\alpha=1$ (or $\alpha=-1$ ) the lattice transformation $\exp \left(i t \hat{\gamma}_{5}\right.$ ) corresponding to eq. (16) is compact, while its continuum counterpart is not.

Infinitesimal translation

In the continuum we have $\delta \psi_{0}(\chi)=\epsilon \hat{\partial}_{\mu} \psi_{0}(\chi), \delta \bar{\psi}_{0}(\bar{\chi})=\epsilon \bar{\psi}_{0}(\bar{\chi}) \hat{\partial}_{\mu}^{\dagger}$, where $\left(\hat{\partial}_{\mu}\right)_{x y}=\partial_{\mu}^{x} \delta(x-y)$. Our general procedure leads to the lattice transformations

$$
\begin{aligned}
\delta \chi & =\epsilon \omega \hat{\partial}_{\mu} \psi_{0}(\chi) \\
\delta \bar{\chi} & =\epsilon \bar{\psi}_{0}(\bar{\chi}) \hat{\partial}_{\mu}^{\dagger} \omega^{\dagger} .
\end{aligned}
$$

Using $\left[D, \hat{\partial}_{\mu}\right]=0$ it is a simple exercise to show explicitly that the lattice action is invariant under this infinitesimal translation.

What was shown above remains valid also in the presence of gauge fields. In the RG approach, eq. (11), the lattice Dirac operator $\mathcal{D}$ lives on some lattice gauge field background $V$, while on the r.h.s. the continuum Dirac operator $D$ and the blocking $\omega$ are defined on a corresponding continuum gauge field, which is obtained from $V$ by a similar minimization procedure involving only gauge fields 12 .

\section{Generalization to interactive theories: the non- linear $\sigma$ model}

We illustrate the generalization of the technique used above on the example of the $d=2$ nonlinear sigma model. The equation analogous to eq. (11) reads in 
this case

$$
\mathcal{A}(\vec{R})=\min _{\{\vec{S}\}}(A(\vec{S})+T(\vec{R}, \omega(\vec{S})))
$$

where $T$ is the block transformation, $\omega(\vec{S})$ defines the averaging, $A(\vec{S})$ is the continuum action, while $\mathcal{A}(\vec{R})$ is the (fixed point [13]) action on the lattice. For the averaging one might take the flat, non-overlapping averaging in eq. (2). A simple example for $T$ is

$$
\begin{aligned}
T & =2 \kappa \sum_{n}\left(\vec{R}_{n}-\frac{(\omega \vec{S})_{n}}{\left|(\omega \vec{S})_{n}\right|}\right)^{2} \\
& =4 \kappa \sum_{n}\left(1-\vec{R}_{n} \tilde{\omega}(\vec{S})_{n}\right)
\end{aligned}
$$

where we introduced the notation

$$
\tilde{\omega}(\vec{S})_{n}=\frac{(\omega \vec{S})_{n}}{\left|(\omega \vec{S})_{n}\right|}, \quad \tilde{\omega}(\vec{S})^{2}=1
$$

For notational simplicity we shall take $4 \kappa=1$. The minimizing field in eq. (20) is denoted by $\vec{S}_{0}=\vec{S}_{0}(\vec{R})$. Consider now an infinitesimal symmetry transformation of the continuum action (infinitesimal translation, for example) acting on the minimizing field $\vec{S}_{0}(\vec{R}) \rightarrow \vec{S}_{0}(\vec{R})+\delta \vec{S}_{0}(\vec{R})$. Introduce the notation

$$
\tilde{\omega}\left(\vec{S}_{0}+\delta \vec{S}_{0}\right)=\tilde{\omega}\left(\vec{S}_{0}\right)+\delta \tilde{\omega}, \quad\left(\delta \tilde{\omega}, \tilde{\omega}\left(\vec{S}_{0}\right)\right)=0 .
$$

Following the procedure used for fermions above, we compensate the change of the blocking term $T$ by changing the lattice configuration $\vec{R} \rightarrow \vec{R}+\delta \vec{R}$

$$
\left(\delta \vec{R}, \tilde{\omega}\left(\vec{S}_{0}(\vec{R})\right)\right)+(\vec{R}, \delta \tilde{\omega})=0, \quad(\vec{R}, \delta \vec{R})=0
$$

where we used eq. (21). The solution of eq. (24) can be written as

$$
\delta \vec{R}=\delta \tilde{\omega}(\tilde{\omega}, \vec{R})-\tilde{\omega}(\delta \tilde{\omega}, \vec{R}) .
$$

If $\delta \vec{S}_{0}(\vec{R})$ is a symmetry transformation in the continuum, then $\mathcal{A}(\vec{R}+\delta \vec{R})=$ $\mathcal{A}(\vec{R})$, i.e. $\delta \vec{R}$ in eq. (25) is a symmetry transformation on the lattice.

Acknowledgements P.H. is indebted for the kind hospitality and for the discussions with many of the participants at the ILFTN Workshop in Nara and at the Workshop at YITP in Kyoto. P.H. and F.N. thank the kind invitation to the Ringberg Meeting where part of this work was presented. This work was supported by the Schweizerischer Nationalfonds. 


\section{References}

[1] P. H. Ginsparg and K. G. Wilson, Phys. Rev. D25 (1982) 2649.

[2] P. Hasenfratz, Nucl. Phys. Proc. Suppl. $63 \quad$ (1998) 53 arXiv:hep-lat/9709110.

[3] H. Neuberger, Phys. Lett. B427 (1998) 353 arXiv:hep-lat/9801031.

[4] Y. Kikukawa, T. Noguchi, arXiv:hep-lat/9902022

[5] M. Lüscher, Phys. Lett. B428 (1998) 342 arXiv:hep-lat/9802011.

[6] W. Bietenholz and U.-J. Wiese, Nucl. Phys. B464 (1996) 319 arXiv:hep-lat/9510026 and references therein.

[7] N. B. Nielsen and M. Ninomiya, Nucl. Phys. B185 (1981) 20.

[8] U.-J. Wiese, Phys. Lett. B315 (1993) 417 arXiv:hep-lat/9306003.

[9] M. Lüscher, Nucl. Phys. B549 (1999) 295 arXiv:hep-lat/9811032; ibid B568 (2000) 162 arXiv:hep-lat/9904009;

M. Lüscher, J. High Energy Phys. 06 (2000) 028 arXiv:hep-lat/0006014; H. Suzuki, J. High Energy Phys. 10 (2000) 039 arXiv:hep-lat/0009036;

Y. Kikukawa and Y. Nakayama, Nucl. Phys. B597 (2001) 519 arXiv:hep-lat/0005015;

M. Lüscher, in 'Erice 2000, Theory and experiment heading for new physics', 41-89, arXiv:hep-th/0102028 and references therein.

[10] M. Lüscher, Nucl. Phys. B538 (1999) 515 arXiv:hep-lat/9808021

R. Narayanan, Phys. Rev. D58 (1998) 97501 arXiv:hep-lat/9802018;

F. Niedermayer, Nucl. Phys. Proc. Suppl. 73 (1999) 105 arXiv:hep-lat/9810026.

[11] P. Hasenfratz, Nucl. Phys. Proc. Suppl. B106 (2002) 159 arXiv:hep-lat/0111023;

K. Fujikawa, M. Ishibashi and H. Suzuki, JHEP 0204 (2002) 046 arXiv:hep-lat/0203016;

Nucl. Phys. Proc. Suppl. B119 (2003) 781, arXiv:hep-lat/0203016;

K. Fujikawa, and H. Suzuki, Phys. Rev. D67 (2003) 034506 arXiv:hep-lat/0210013;

P. Hasenfratz and M. Bissegger, Phys. Lett. B613 (2005) 57 arXiv:hep-lat/0501010.

[12] T. DeGrand, A. Hasenfratz, P. Hasenfratz and F. Niedermayer, Nucl. Phys. B454 (1995) 587 arXiv:hep-lat/9506030.

[13] P. Hasenfratz and F. Niedermayer, Nucl. Phys. B414 (1994) 785 arXiv:hep-lat/9308004. 